\title{
FEASIBILITY TEST OF A FAST MICRO GAS CHROMATOGRAPHY SYSTEM USING FIVE MICROVALVES AND A MICROCOLUMN \\ Byunghoon Bae ${ }^{1,2}$, Adarsh D. Radadia ${ }^{2}$, Jeahyeong Han ${ }^{3}$, Junghoon Yeom ${ }^{3}$, Richard I. Masel ${ }^{1,2}$, and Mark A. Shannon ${ }^{1,3}$ \\ ${ }^{1}$ Cbana Laboratories, Inc., Champaign, IL 61820 \\ ${ }^{2}$ Department of Chemical and Biomolecular Engineering, University of Illinois, Urbana, IL 61801 \\ ${ }^{3}$ Department of Mechanical Science and Engineering, University of Illinois, Urbana, IL, 61801
}

\begin{abstract}
We present a new type of a fast MEMS Gas Chromatography (GC) system that integrates five microvalves and microcolumn for rapid detection of species for a flame ionization detector (FID). The integrated GC samples mixed-gas species, preconcentrates them, and injects a sharp band into the microcolumn with $50 \mu$ s pulse width. The microcolumn can then separate and detect 8 components in the species within 10 seconds with 25 psi of the carrier gas. The fast speed of this GC is enabled by microsecond switching microvalve, low dispersion design, and a new coating technique on the microcolumn.
\end{abstract}

\section{INTRODUCTION}

Many efforts have been made to build fast and portable micro-GC systems for various applications. Homeland security and many on-site industrial applications need fully integrated micro-GC systems with injector microvalves, preconcentrator (PC), microcolumn, detector, and a micropump for sampling. However, no fully integrated system has been developed yet. MEMS PCs including [1] have been published but without integration of other components. A micropump-driven GC system was developed by Kim et al. [2], but their system still employs a separate injection system, lowering GC speed and resolution. Microvalves integrated with a PC was reported by Bae et al. [3].

In this paper, we present a new type of a fast MEMS GC system that integrates five microvalves and microcolumn for rapid detection of species for an FID.

\section{SYSTEM CONFIGURATION}

Figure 1 shows a schematic diagram and device pictures of five microvalves and a microcolumn. Figure 2 shows photographs of all the layers of the microvalves and how they are stacked. To achieve fast operation, the five microvalves employs electrostatic actuation, and each valve can switch states in 50 microseconds [4]. The five integrated microvalves consist of two microvalves to load the sample gas, and three microvales to route and inject the sweep gas through a one- $\mu \mathrm{L}$ chamber to the microcolumn that is designed for low dispersion and has a new deactivation method applied. The microcolumn has $34 \mathrm{~cm}$-long serpentine channels whose cross-section is $100 \times 100 \mu \mathrm{m}^{2}$ with a design generates the lower dispersion than spiral [5]. The integrated GC samples mixed-gas species, preconcentrates them, and injects a sharp band into the microcolumn with $50 \mu$ s pulse width. The microcolumn can then separate and detect 8 components in the species within 10 seconds with 25 psi of the carrier gas. The fast speed of this GC is enabled by MEMS sizing and fabrication, microsecond switching microvalve, low dispersion design, and a new coating technique on the microcolumn, which enables the detection of poisonous gases, toxic industrial compounds, and other volatile compounds to be separated in seconds, rather than tens of minutes with conventional GC systems.

\section{RESULTS}

Figure 3 shows a separation of 8 chemical components using a standalone microcolumn with $25 \mathrm{psi}_{2}$ carrier gas and temperature setting of $90 \sim 98^{\circ} \mathrm{C}$. As an injected plug moves down the column, the plug experiences broadening due to diffusion, velocity-induced shearing, and smearing at bends. Figure 4 shows toluene peaks through the same microcolumn as the one used in the previous experiment. Toluene vapor is sampled at ambient temperature and injected every 10 seconds by the microvalves following the sequence in Fig. 1a to the microcolumn The $\mathrm{H}_{2}$ sweep gas is 3 psi,

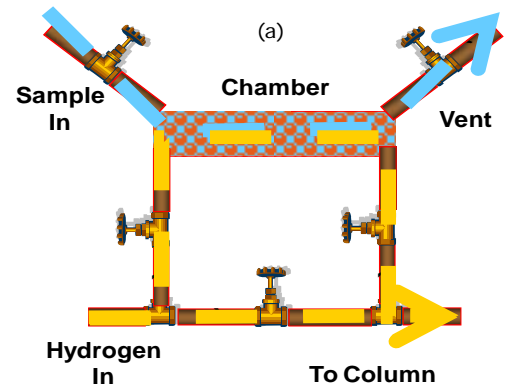

(b)

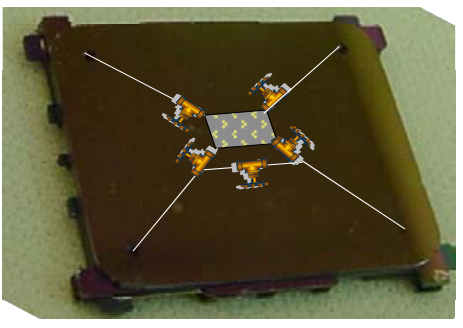

(d)

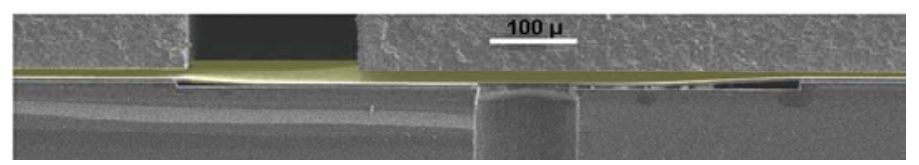

(c)
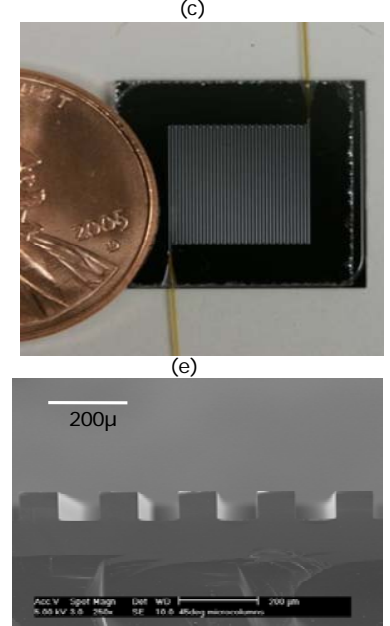

Figure 1: (a) A schematic diagram shows how all the five valves are used for sampling gas (blue line) and for sweeping the sample through to the column with $\mathrm{H}_{2}$ (yellow line); device pictures of (b) five microvalves and (c) microcolumn; and SEM images of the cross-sections of (d) a microvalve and (e) the microcolumns. 


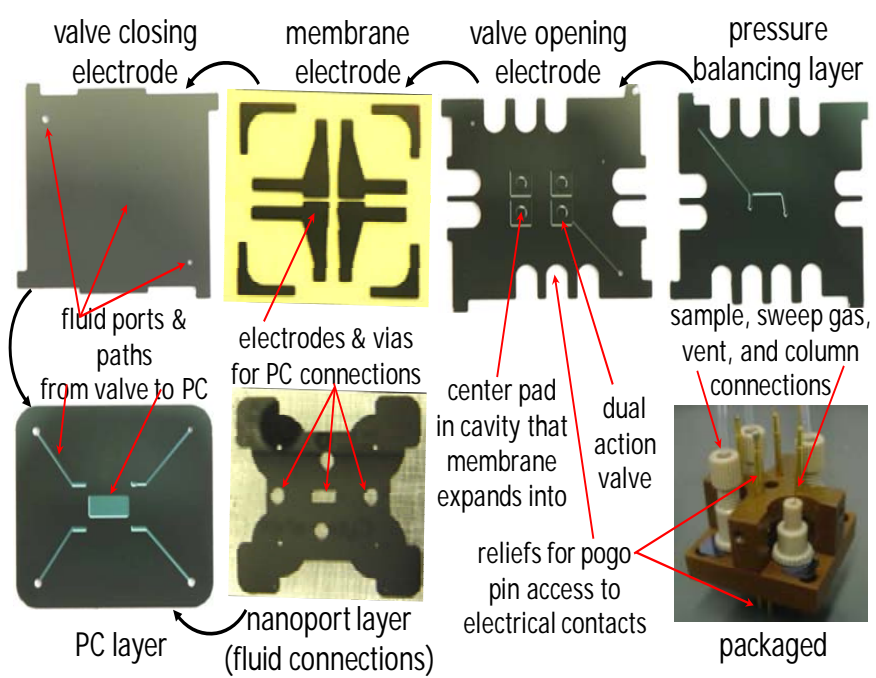

Figure 2: Photos of all the layers of the microvalves and how they are stacked.

which is more than 8 times less than the pressure used for the standalone column in Fig. 3. Figure 4 (b) shows that the $100 \mathrm{~ms}$ width peaks are as sharp as and slightly faster than the peak from the standalone microcolumn. This result is attributed to the microsecond switching performance of the microvalves in addition to the design of the low dispersion microcolumn. To integrate the five microvalves and microcolumn, a connecting layer was fabricated that not only connects the five microvalves to the microcolumn, but also has inlet and outlet fluidic ports where Nanoports ${ }^{\circledR}$ are used as shown in Fig. 5.

\section{CONCLUSIONS}

The feasibility of a micro-GC system that uses five fast-acting microvales valves and a microcolumn has been shown through

(a)

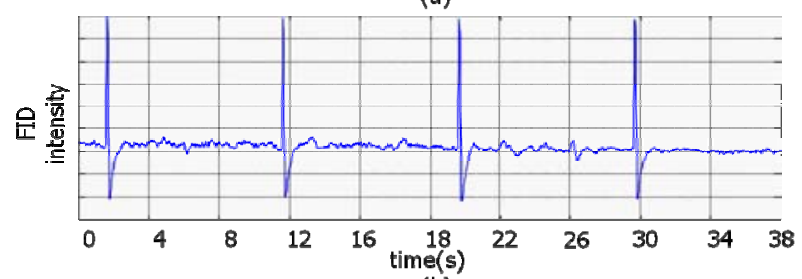

(b)

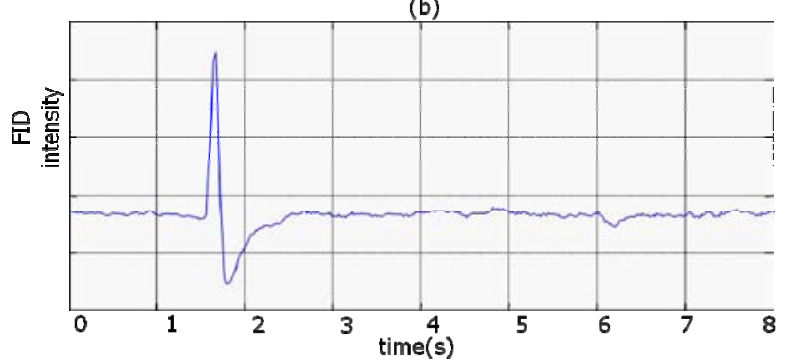

Figure 4: Toluene peaks through the same microcolumn as the one used in the previous experiment. (a) Toluene vapor at ambient temperature being sampled and injected every 10 second by five microvalves to the microcolumn via a capillary, with $\mathrm{H}_{2}$ carrier gas at 3 psi, which is more than 8 times less than the pressure used for the standalone column. (b) The $100 \mathrm{~ms}$ width peaks are as sharp as and slightly faster than the peak from the standalone microcolumn. This result is attributed to microsecond switching performance of the microvalves in addition to the design of the low dispersion microcolumn.

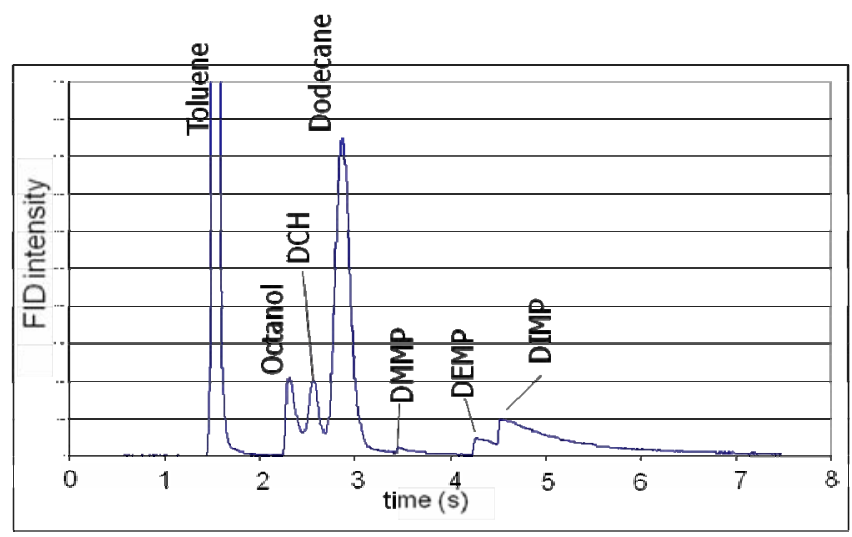

Figure 3: A separation of 8 chemical components using a standalone microcolumn with 25 psi $\mathrm{H}_{2}$ carrier gas at a temperature between $90 \sim 98^{\circ} \mathrm{C}$. As an injected plug moves down the column, the plug experiences broadening due to diffusion, velocity-induced shearing, and smearing at bends.

simple testing. The peak width will be even sharper by removing a dead volume between components that increases band broadening, allowing higher pressures to be used, and thereby reducing analysis time further.

\section{ACKNOWLEDGEMENTS}

We gratefully acknowledge financial support from the Defense Advanced Research Projects Agency (DARPA) under U.S.Air Force grant FA8650-04-1-7121. Any opinions, findings, and conclusions or recommendations expressed in this manuscript are those of the authors and do not necessarily reflect the views of the Defense Advanced Projects Research Agency or the U.S. Air Force.

\section{REFERENCES}

[1] W.C. Tian, H.K.L. Chan, C.J. Lu, S.W. Pang and E.T. Zellers, "Multiple-Stage Microfabricated Preconcentrator-Focuser for Micro Gas Chromatography System,” Journal of Microelectromechanical Systems, Vol. 14, No. 3, pp 498-507. 2005. [2] H. Kim, W. H. Steinecker, S. H. Reidy, G. R. Lambertus, A. A. Astle, K. Najafi, E. T. Zellers, L. P. Bernal, P. D. Washabaugh, K. D.Wise, "A Micropump-Driven High-Speed MEMS Gas Chromatography System,” Transducers 07’, pp. 1505-1508, 2007. [3] B. Bae, J. Yeom, R. I. Masel, M. A. Shannon, "A Five-Microvalve Fully Integrated Preconcentrator,” IEEE Sensors conference, pp. 1353-1356, 2007.

[4] B. Bae, J. Han, R. I. Masel, M. A. Shannon, "A Bidirectional Electrostatic Microvalve With Microsecond Switching Performance," Journal of Microelectromechanical Systems, Vol. 16, No. 6, pp.1461-1471, 2007.

[5] A. D. Radadia, R. I. Masel, and M. A. Shannon, "New Colums Design for MicroGC,” Transducers 07’, pp. 2011-2014, 2007.
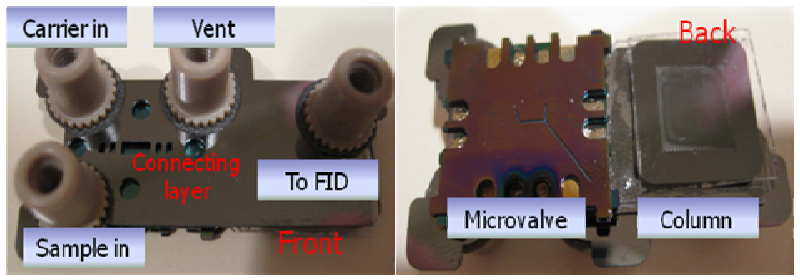

Figure 5: A photo of the five microvalves integrated with the microcolumn. 